\title{
Nanostructure and Optical Properties of Silver Helical Pentagon Nanosculptured Thin Films
}

\author{
Hadi Savaloni, Fatemh Haydari-Nasab, and Mariam Malmir \\ Department of Physics, University of Tehran, North Kargar Street, Tehran, Iran \\ Correspondence should be addressed to Hadi Savaloni; savaloni@khayam.ut.ac.ir
}

Received 20 May 2013; Revised 12 October 2013; Accepted 31 October 2013; Published 20 January 2014

Academic Editor: Michael C. Tringides

Copyright (c) 2014 Hadi Savaloni et al. This is an open access article distributed under the Creative Commons Attribution License, which permits unrestricted use, distribution, and reproduction in any medium, provided the original work is properly cited.

\begin{abstract}
Silver helical pentagon shaped nanosculptured thin films (HPNSTFs) were produced using oblique angle deposition method in conjunction with the rotation of sample holder under controlled conditions. The $s$-polarization extinction spectra obtained at different azimuthal angles $(\varphi)$ and low incidence angle (i.e., $10^{\circ}$ ) from the Ag (HPNSTF) did not show significant change in the plasmon peak position, while at higher incidence angle (i.e., $70^{\circ}$ ) a blue shift appeared for the broad peak which was observed for lower incidence angle (i.e., $10^{\circ}$ ) and occurred at lower wavelength. In case of $p$-polarized light a very broad peak was obtained for the $70^{\circ}$ incidence angle and for different $\varphi$ angles and when compared with the lower incidence angle results it can be concluded that it is gone under a red shift. Polar diagrams of the samples showed slight anisotropy that should be due to high symmetry of the pentagon helical structure.
\end{abstract}

\section{Introduction}

Sculptured thin films (STFs) are columnar (range between 1 and $100 \mathrm{~nm}$ ) thin films deposited on substrate with controlled azimuthal rotation, $\varphi$, and tilt angle, $\alpha$, by a method known as oblique angle deposition (OAD) $\left(\alpha<85^{\circ}\right)$ or glancing angle deposition (GLAD) $\left(\alpha>85^{\circ}\right)$ [1]. The structure of STFs consists of rough surface with a much higher void fraction than the films produced using conventional deposition method (i.e., deposition at normal vapor incident angle). Hence, these nanostructures especially the metallic nanostructures with specific properties dependent on their size have created an interesting research environment for scientists in different fields of application like biomedicine [2-6], antibacterial [7], optics [8-11], and other fields of science. Polygonal helical sculptured thin films have been subject to investigation during the last few years. Hodgkinson was first to report on these films in 2004 [12] theoretically and experimentally, then Van Popta et al. [13] reported on double-handed circular Bragg phenomena in polygonal helix thin films and the next major advance was made by Dixit and Lakhtakia [14]. Kennedy et al. [15] by using GLAD technique fabricated tetragonal square spiral photonic crystals. Optical properties of square gold nanospirals have been investigated numerically by Abdeddaim et al. [16]. Robbie and Brett [17] produced sculptured thin films of different shapes (e.g., slanted nanorods, zigzag, and helices) using the GLAD technique.

Experiments have demonstrated that the plasmon peak depends strongly on the morphology and assembly of the nanoparticles, such as diameter [18], aspect ratio [19], shape [20], or assembly of array and the lattice parameters of the array [21] and the dielectric constant of the host medium [2224] as well as the wavelength of the incident light. Therefore, in order to control and adjust the surface plasmon resonance wavelength, it is required to use a method for production of a nanostructure that will provide us with a proper control of the above-mentioned parameters. Nanostructures with more structural complexity may provide higher number of plasmonic peaks. Nehl et al. [25] have reported on the plasmonic peaks of star shaped gold nanostructures and observed that the plasmon resonances are stronger at sharp tips (points) and are strongly dependent on the polarization of the incident light.

Chaney et al. [26] used glancing angle deposition to produce three-dimensional structures that enhance the localized 


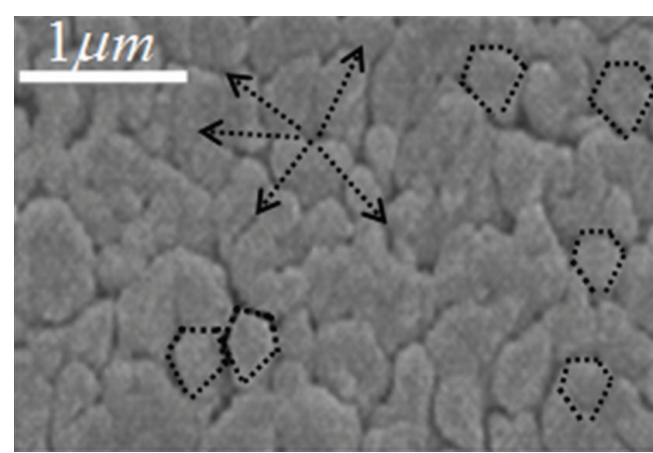

(a)

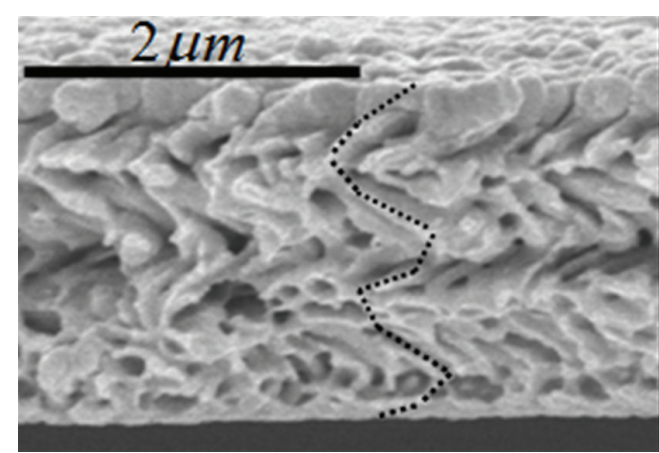

(b)

FIGURE 1: FESEM images of silver chiral pentagon sculptured thin film; (a) surface, (b) cross-section.

surface plasmon resonances (LSPR). Zhang and Zhao [27, 28] have also carried out an extensive work on the descript dipole approximation (DDA) to obtain the extinction spectra of sculptured nanostructures.

In this work, we report on the nanostructure and optical properties of pentagon helical nanosculptured silver thin films produced using oblique angle deposition technique together with the controlled rotation of substrate. In order to investigate the optical behavior of the pentagon helical films both $s$ - and $p$-polarized lights at different incidence angles and azimuthal angles were used.

\section{Experimental Details}

Silver (99.99\% purity) helical pentagon shaped nanosculptured thin films were deposited at $75^{\circ}$ oblique angle deposition on glass $\left(15 \times 15 \mathrm{~mm}^{2}\right.$ microscope slide) substrates, using resistive evaporation from tungsten boats with an outlet of $6 \mathrm{~mm}$ diameter at room temperature. An Edwards (Edwards E19 A3) coating plant with a base pressure of $3 \times 10^{-7} \mathrm{mbar}$ and a deposition rate of $2.5 \AA \mathrm{s}^{-1}$ was used. In order to achieve a uniform deposition on the substrates a $30 \mathrm{~cm}$ distance between the evaporation source and the substrate was chosen. In this arrangement the vapor source (6 $\mathrm{mm}$ in diameter) behaves like a point source with a cosine distribution and because of $30 \mathrm{~cm}$ distance between the vapor source and the substrate it is expected that vapor has a straight trajectories (i.e., no appreciable scattering due to the large mean free path $\left(\sim 10^{3}-10^{4} \mathrm{~cm}[29]\right)$ occurs). Optical reflection from the reproduced samples was agreed to be within $5 \%$. Different mechanical movements of substrate holder are controlled via interface to a computer in which the related software is written and installed [30].

In order to produce the helical pentagon shaped thin film the deposition of silver was carried out from a preset/initial substrate position for formation of one side/arm of the pentagon and then the process was repeated by successive rotation of the substrate holder by $72^{\circ}$ in the anticlockwise direction for ten times. Therefore, the pentagon helical structures with 2 pitches were produced. The length of each arm deposited arranged to be about $350 \mathrm{~nm}$. It is worthwhile to clarify that this is the length of each arm and not the height of the arm. Considering that the deposition of the film was at $75^{\circ}$ to the substrate surface normal, using the Tait [31] rule which is more agreeable with the experimental data at deposition angles greater than $60^{\circ}$ rather than the tangent rule $[32,33]$ as

$$
\beta=\alpha-\sin ^{-1}\left(\frac{1-\cos \alpha}{2}\right)
$$

we get a growth angle of $53.2^{\circ}$ which is with respect to the surface normal. Hence, the height of each arm of the pentagon can be calculated as $350 \times \sin (90-53.2)=209 \mathrm{~nm}$. Therefore, the total height of the helical pentagon produced in this work with ten arms should be equal to $\sim 2.1$ micrometers. This in fact is very close to the measurement from the crosssection of the film in Figure 1.

All substrates were ultrasonically cleaned in heated acetone then ethanol before being mounted on the substrate holder for deposition. The surface roughness of the substrates was measured by a Talysurf profilometer and AFM and the rms substrate surface roughness $R_{q}$ obtained using these methods was $0.3 \mathrm{~nm}$ and $0.9 \mathrm{~nm}$, respectively.

The deposition rate was measured by a quartz crystal deposition rate controller (Sigma Instruments, SQM-160, USA) positioned close to the substrate and at almost the same azimuthal angle as that of the substrate. This was corrected after obtaining the film thickness using field emission electron microscope (FESEM; Hitachi S-4100 SEM, Japan). FESEM samples were coated with a very thin layer of gold to prevent the charging effect. The surface physical morphology and roughness was obtained by means of atomic force microscope (AFM; NT-MDT SOLVER, with a Si tip of $10 \mathrm{~nm}$ radius in contact mode) analysis. Reflection and transmittance spectra of the samples were obtained using a single beam spectrophotometer (Aquila nkd-8000) in the spectral range of $350-1050 \mathrm{~nm}$ using both $s$ - and $p$ polarization measurements in steps of $5 \mathrm{~nm}$ wavelength at different incident angles.

\section{Results and Discussions}

3.1. FESEM and AFM (Nanostructure). FESEM images of helical pentagon shaped sculptured thin films are given 
TABLE 1: Average surface roughness and grain size for helical pentagon silver sculptured thin films.

\begin{tabular}{lcccc}
\hline Ag/glass & $\begin{array}{c}R_{\mathrm{ave}} \\
(\mathrm{nm})\end{array}$ & $\begin{array}{c}R_{\mathrm{rms}} \\
(\mathrm{nm})\end{array}$ & $\begin{array}{c}\text { Diameter of } \\
\text { each arm } \\
(\mathrm{nm})\end{array}$ & $\begin{array}{c}\text { Grain/side } \\
\text { size }(\mathrm{nm})\end{array}$ \\
\hline $\begin{array}{l}\text { Helical } \\
\text { pentagon }\end{array}$ & 16 & 20 & 80 & 244 \\
\hline
\end{tabular}

in Figure 1. The surface structure of pentagon thin film is given in Figure 1(a). Figure 1(b) shows the cross-section of silver helical pentagon shaped thin film. Cross-section of the films was obtained by fracturing the substrate that caused braking of the pitches/arms of the helical pentagon too. Hence, in Figure 1(b) a proper cross-section of pentagon thin films may not be clearly distinguished though a more careful examination of this figure and its comparison with the simulated similar pentagon in Figure 3 confirms the growth of pentagon sculptured thin film. The symmetry of the grown helical pentagons on the surface of the film is shown by geometrical shape (i.e., pentagon) while the orientation of grown arms is also shown by black arrows. These arrows show the principle axes that the columns grow along their directions. With regard to this observation we should also point out the high surface diffusion of silver adatoms, the rise of substrate temperature from its initial setting during almost 5 hours of deposition, and that these films were grown on bare (unseeded) glass substrates. Therefore, all these parameters/effects prevent the pentagon thin films from growing in a perfect ordered structure. However, we may claim that this structure is more applicable for industrial or applied works as preseeding is a costly procedure. The diameter of the vertical columns/helixes including the width of pentagon sides may be estimated as $\sim 350 \mathrm{~nm}$ (the same as length of each arm deposited and mentioned in Section 2) while the diameter of each arm is about $\sim 80 \mathrm{~nm}$.

In Figure 2, 2D and 3D AFM images of silver helical pentagon sculptured shaped thin films are given. Both 2D and 3D images of helical pentagon sculptured thin film clearly show the shape of grown pentagon. Size distributions of the grains of these sculptured thin films were obtained from the 2D AFM images using JMicroVision Code and are given in Table 1. In Table 1 the mean and the root mean square (rms) surface roughness and the average grain size for pentagon sculptured silver thin films are given.

Pentagon and other shaped sculptured nanostructures which consist of high number of corners and recesses (bends) may excite a strong local electromagnetic field (hot spot (HS)) which are responsible for surface enhanced Raman spectroscopy [34]. Savaloni and Babaei [35] produced silver chiral nanoflower shaped structures with different symmetries and obtained a high degree of enhancement of the main bands at 1610,1297 , and $1009 \mathrm{~cm}^{-1}$ that can be assigned to the $C=C$ stretching mode, aromatic ring, stretching ring and in-plane ring mode of $4,4^{\prime}$-bipyridine.

In particular, $\mathrm{Ag}$ and $\mathrm{Au}$ have shown a great ability to generate localized surface plasmon resonance [36]. They may
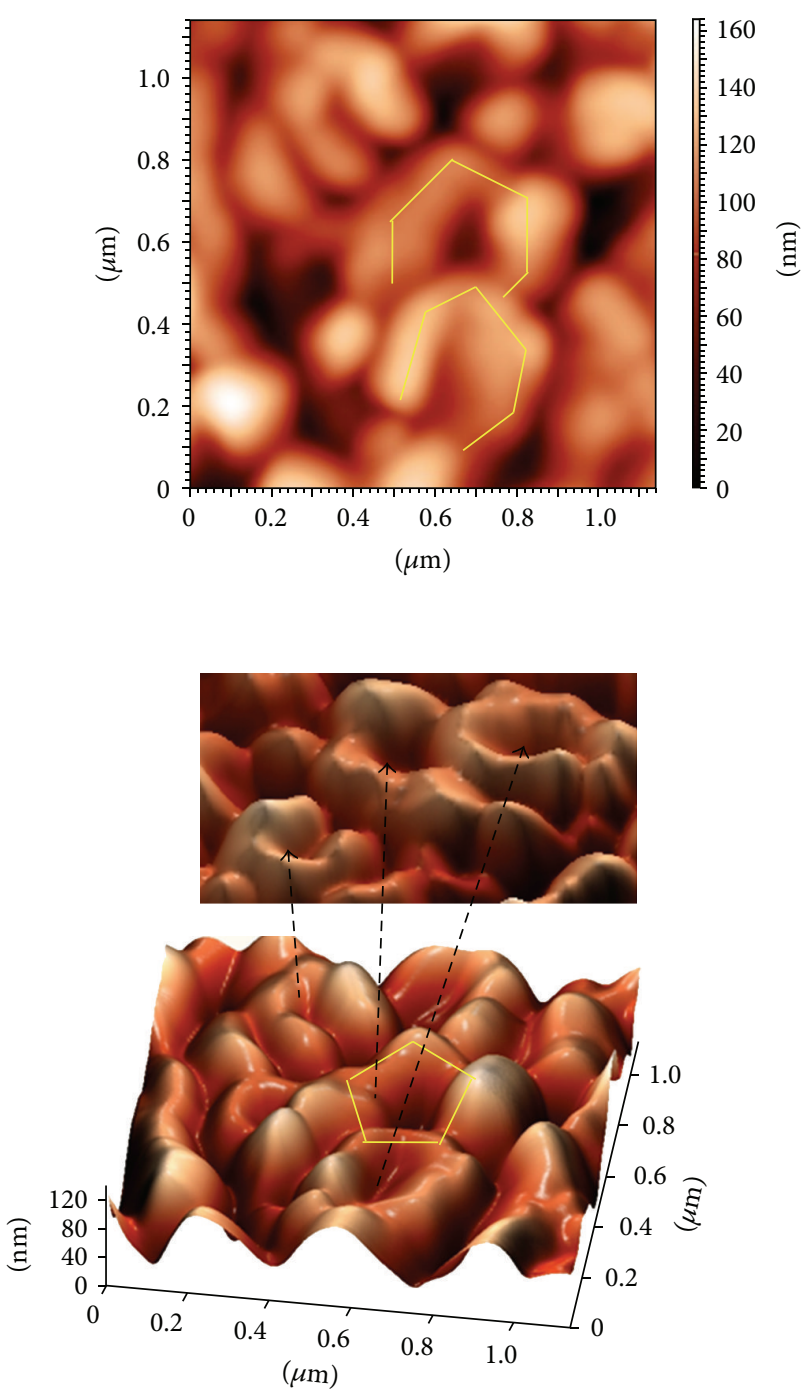

FIGURE 2: 2D and 3D AFM images of the silver chiral pentagon sculptured thin film.

also be used as antibacterial and antiviral agents with much higher activity than ordinary thin films.

3.2. Optical Characteristics. Optical spectra of silver helical pentagon shaped nanosculptured thin films were measured in the wavelength region of 350 to $1050 \mathrm{~nm}$ for both $s$ - and $p$-polarizations and incident light angles of $10^{\circ}$ and $70^{\circ}$ at different azimuthal angles $(\varphi)$ of $0^{\circ}, 45^{\circ}, 90^{\circ}, 135^{\circ}$, and $180^{\circ}$ for each incident angle. Figure 3 shows the schematic of the measurement set up. We define the p-polarization at the plane of grown helical pentagons and the $s$-polarization at normal to this plane (Figure 3).

It is well established both theoretically and experimentally that a sufficiently thick columnar thin film has a negligible transmittance [37] which is also proven for helical sculptured thin films by Lakhtakia [38]. Hence, we may use $(1-R)$ as a good approximated value for film extinction. SiabiGarjan and Savaloni [39] also by using the homogenization theory have shown that the optical response of metallic 


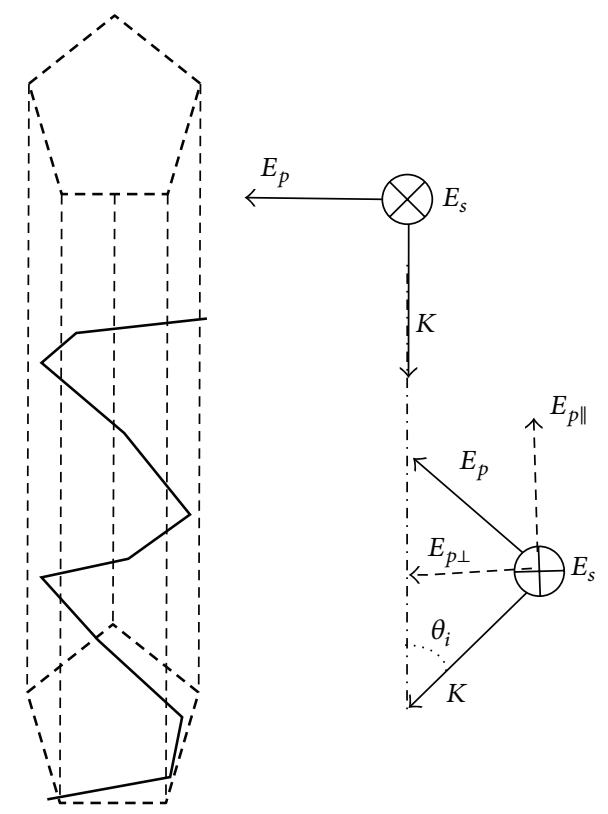

FIGURE 3: Schematic of chiral pentagon growth and the definition of the incident polarization directions and decomposition of the $p$ polarized field by changing the incident light direction.

structures is affected by a limited thickness of thin film $(\sim 320 \mathrm{~nm})$ and further increase of the film thickness has no effect on the optical spectra. Therefore, it may be suggested that the optical results discussed in this paper are related to the top $320 \mathrm{~nm}$ thickness of the film. Considering that the height of each arm is about $209 \mathrm{~nm}$ we may suggest that the top one and a half arm of the helical pentagon sculptured thin film produced in this work may be responsible for the optical responses discussed in this section. Then one may ask why we have produced pentagon thin films with ten arms (two pitches). This was because of two reasons: (a) thickness of conventional sculptured thin films is usually in the range of microns (this is required to observe the formation of the designed structure) $[40,41]$ and because of the formation of the bottom layer $[30,42]$ in these films one may not be sure that the first arm is acting as the actual arm of the sculptured structure; (b) in order to obtain the structural characteristics such as FESEM and AFM discussed in the preceding sections.

If the feature size is about the same or greater than the wavelength of the incident light then scattering becomes the dominant process in the extinction spectrum (i.e., absorption + scattering) [43]. Hence, according to the AFM results (Table 1) since the size of the grains on these films is smaller than or just about the minimum wavelength (i.e., $350 \mathrm{~nm}$ ) it may be concluded that the scattering contribution in our work is negligible.

3.2.1. Optical Spectra of Helical Pentagon Silver Sculptured Thin Film. As it is illustrated in Figure 3 when the incident light interacts with the helical pentagon at an angle the $E_{p}$ field decomposes into two components: a field parallel $E_{p \|}$ to and a field perpendicular $E_{p \perp}$ to the long axis of the pentagon. Therefore, the larger $\theta_{i}$ results in larger $E_{p \|}$ component (Figure 3). Therefore, the results of low incidence angle $\left(10^{\circ}\right)$ should contain information about the surface morphology of the film, including anisotropy when the $\varphi$ angle is changed, while high incidence angle $\left(70^{\circ}\right)$ interacts with the Ag column with its maximum electric field. With regard to $s$-polarization, when the $\varphi$ angle is changed, because of surface anisotropy of pentagon, we should obtain some information about the surface morphology.

Since the symmetry along the helical axis of the pentagon sculptured thin film (fivefold symmetry) is not the same as circular chiral sculptured thin films [36] (maximum symmetry) during optical measurements at different azimuthal angles, one may expect a change in the peak position which can be due to the changes of the parallel and perpendicular components of the incident light on the pentagon arms (that takes different orientations with respect to the direction of the incident light components).

As mentioned before our substrates were not preseeded; therefore, the samples produced in this work consist of an assembly of randomly distributed helical pentagons with some imperfections. Hence, one should expect a difference between our experimental results and those obtained from theoretical studies such as discrete dipole approximation (DDA) though there has been no such report on helical pentagon silver sculptured thin films so far. We have studied this aspect of work and our final results will be published in the near future, while our work [39] on the extinction spectra and electric field enhancement of silver chiral nanoflower shaped nanoparticle, comparing the results of discrete dipole approximation with experimental results [44-46], has shown that the experimental spectra are the result of the overlap of the extinction spectra of chiral nanoflower nanoparticles whose structure may be of different size, orientation, random distribution, and in some cases incomplete form of a chiral nanoflower. A somewhat similar model may be used for prediction of results for predesigned devices in different applications. In general, we may emphasize that the results presented in this work may present the required expected applied properties.

Figures 4(a) and 4(b) show the (1-R) spectra of the helical pentagon for $p$-and $s$-polarizations measured at $10^{\circ}$ incidence angle and different $\varphi$ angles (ranging from $0^{\circ}$ to $180^{\circ}$ in steps of $45^{\circ}$ ), respectively.

At low incidence angles (e.g., $10^{\circ}$ ) we may speculate the presence of a peak at about $\sim 350 \mathrm{~nm}$ (because of our limitation for the measurement at the lower wavelengths) and observe a broad peak at longer wavelengths for both $s$ - and $p$-polarized lights. Details of the position of these peaks are summarized in Table 2.

Figures 4(c) and 4(d) similarly show the $(1-R)$ spectra for both $p$-and $s$-polarizations measured at $70^{\circ}$ incidence angle, respectively. It can be observed that these spectra are different from those in Figures 4(a) and 4(b). With regard to the spectrum obtained for $s$-polarized light, it may be suggested that the broad peak which was observed for lower incidence angle (i.e., $10^{\circ}$ ) has gone under a blue shift and occurred at lower wavelength. The position of this peak for different $\varphi$ angles is also given in Table 2 that can be compared with those obtained from the lower incidence angle of $10^{\circ}$. A very broad 


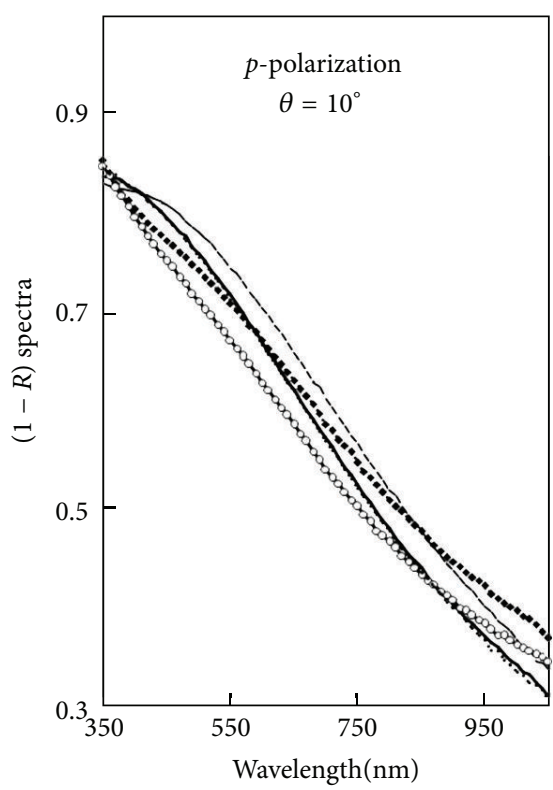

(a)

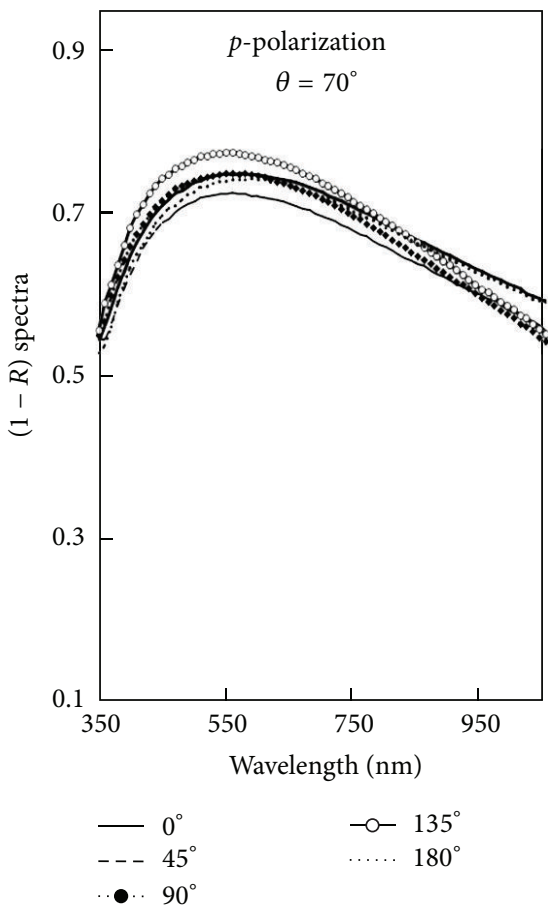

(c)

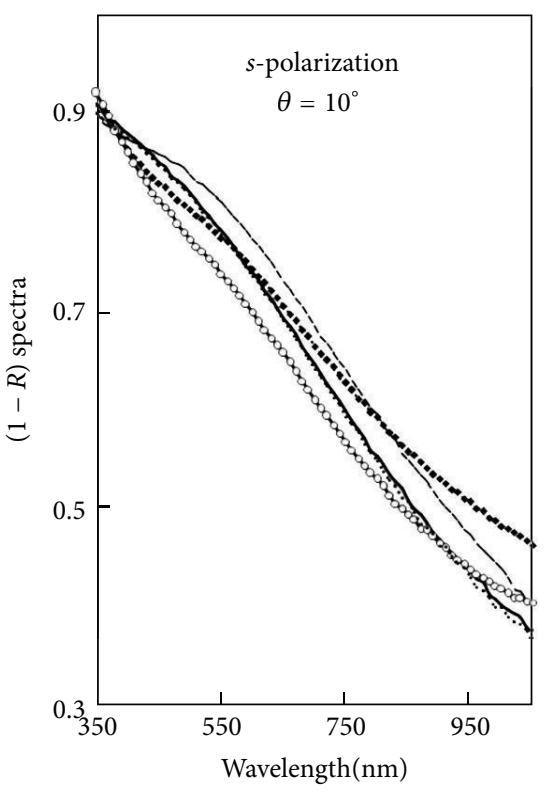

(b)

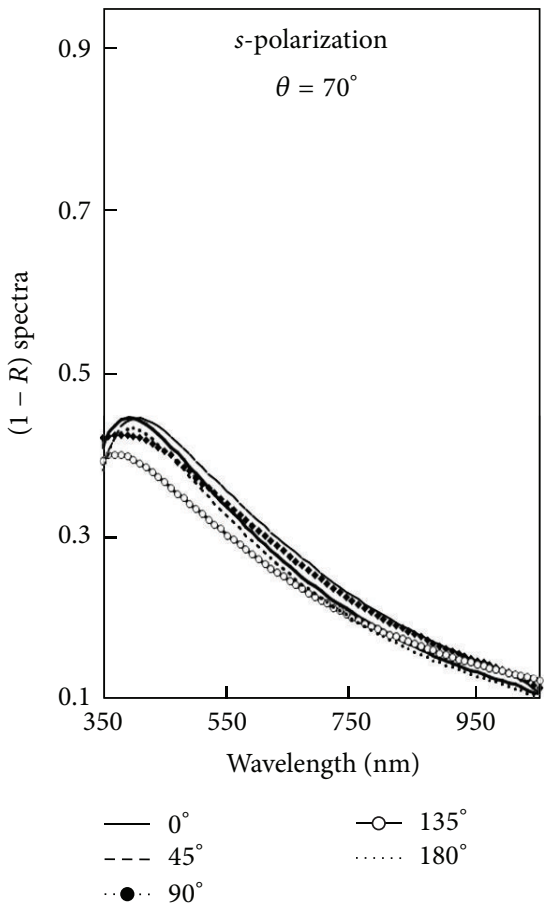

(d)

FIGURE 4: $(1-R)$ spectra of Ag chiral pentagon thin film obtained at two different incident angles $(\theta)$ and different azimuthal angles. ((a) and (b) ) $\theta=10^{\circ}, p$ - and $s$-polarizations, respectively; ((c) and (d)) $\theta=70^{\circ}, p$ - and $s$-polarizations, respectively.

peak is obtained for $p$-polarized light (Figure 4(c)). When the position of this peak for different $\varphi$ angles is compared with those obtained for low incidence angle it can be deduced that it is gone under a red shift, while there is not any sign of the peak at about $350 \mathrm{~nm}$ wavelength. This is the result of increased $E_{p \|}$ component of the $p$-polarized light at this angle of incidence.
We should also mention that the helical pentagons grown on our substrates suffer from a degree of misalignment which can be seen in the FESEM images, though a large number of them are aligned in one direction. Hence, in general there is a distribution. Therefore, since at $70^{\circ}$ incident angle the $E_{p \|}$ is stronger the broad peak in Figure 4(c) is observed. In fact the broad width of this peak can be due to the excitation 
TABLE 2: Details of the absorbance $(1-R)$ peak wavelength and intensity for both $s$-and $p$-polarization extinction spectra of helical pentagon sculptured thin film.

\begin{tabular}{|c|c|c|c|c|c|}
\hline $\begin{array}{l}\text { Azimuthal } \\
\text { angle }\left(^{\circ}\right)\end{array}$ & $\begin{array}{l}\text { Incidence } \\
\text { angle }\left(^{\circ}\right)\end{array}$ & $\begin{array}{c}s \text {-polarization } \\
\text { (peak position) } \\
(\mathrm{nm})\end{array}$ & $\begin{array}{c}s \text {-polarization } \\
\text { (intensity) }\end{array}$ & $\begin{array}{c}\text { p-polarization } \\
\text { (peak position) } \\
(\mathrm{nm})\end{array}$ & $\begin{array}{l}p \text {-polarization } \\
\text { (intensity) }\end{array}$ \\
\hline 0 & 10 & 370 & 0.880 & 370 & 0.886 \\
\hline 45 & 10 & 360 & 0.886 & 370 & 0.890 \\
\hline 90 & 10 & 370 & 0.888 & 460 & 0.843 \\
\hline 135 & 10 & 370 & 0.892 & 460 & 0.858 \\
\hline 180 & 10 & 390 & 0.876 & 450 & 0.821 \\
\hline 0 & 70 & 390 & 0.445 & 520 & 0.743 \\
\hline 45 & 70 & 390 & 0.441 & 520 & 0.721 \\
\hline 90 & 70 & 360 & 0.422 & 540 & 0.747 \\
\hline 135 & 70 & 360 & 0.402 & 540 & 0.773 \\
\hline 180 & 70 & 390 & 0.431 & 540 & 0.741 \\
\hline
\end{tabular}

of a few plasmon modes (overlap of few peaks) because of the distribution of pentagons on the substrate surface as discussed above.

If the results of $s$-polarization for incident angle of $10^{\circ}$ in Figure 4(b) are compared it can be observed that not only there is a difference in the position of the more obvious peak (about 400-550 $\mathrm{nm}$ ) but also the order of their intensity with regard to the $\varphi$ angle is also varied as follows.

For $10^{\circ}$ incident angles the order is $45^{\circ}, 0^{\circ}$ and $180^{\circ}, 90^{\circ}$, and $135^{\circ}$.

This shows that, since spectra of $0^{\circ}$ and $180^{\circ}$ coincide, our pentagons have almost formed with symmetry near to a chiral shape column.

Almost the same order of variation is also obtained for the $70^{\circ}$ incident angle, where only at the region of the peak, spectra of $0^{\circ}$ and $180^{\circ}$ slightly differ and the peak of $180^{\circ}$ is higher than $0^{\circ}$.

These observations, although, show that films may be isotropic but still the degree of anisotropy must be quantified in the morphology of our silver helical pentagon sculptured thin film, and as mentioned above by azimuthal rotation of the substrate the amount and direction of the film material responsible for light extinction are changed (i.e., it may consist of a full arm or combination of adjoining arms with different partial contribution, considering, as mentioned before, that the incident light can only be affected by the top $320 \mathrm{~nm}$ thickness of the film) which in turn is also responsible for the shift of the peak to higher or lower wavelengths (Table 2). In order to further investigate this issue the polarization dependent optical spectra of these films is discussed in the next section.

With regard to the reduction of the intensity by increasing the incident angle in the extinction spectra obtained from $s$-polarized light (Figures 4(b) and 4(d)) we may refer to the following points in order to explain this observation. (a) Siabi-Garjan et al. [47] investigated the optical properties of $\mathrm{ZnS}$ nanorods and chiral sculptured thin films and observed both experimentally and theoretically the same effect and concluded that by increasing the incidence light angle the reflectance of $p$-polarized light decreases and its extinction increases. The variation of the $s$-polarized light with the incident light angle is opposite to that of $p$-polarized light. This is due to approach of the incidence light angle to the Brewster angle $[48,49]$ which is about 74.6 for the bulk silver sample and wavelength of $600 \mathrm{~nm}$ [50]. Hence, our measurement at $70^{\circ}$ incident light angle was very close to the Brewster angle and for $s$-polarized light we measured high reflectance which decreases the extinction. (b) In other words, as mentioned before, results of Siabi-Garjan and Savaloni [39] in which their investigation, using the homogenization theory, although obtained for perfect arrangement of structures have shown that the optical response of metallic structures is affected by a limited thickness of sculptured thin films $(\sim 320 \mathrm{~nm})$ and further increase of the film thickness has no effect on the optical spectra. Hence, considering that the height of each arm of the helical pentagon in this work is about $210 \mathrm{~nm}$, at low incident angle the $s$-polarized light may interact with a complete arm and a section of underlying arm as well as the bend joining these two arms (hot spot). However, when the incident angle is increased the $s$-polarized light may not interact with the underlying arm and may only interact with the top surface of the film (missing the hot spot). This will decrease the intensity of the extinction spectra. In other words, with increasing the incident angle the number of dipoles that interact with the incident $s$-polarized light decreases.

The blue shift observed in these results can also be explained by adapting the above discussion in the form that by reduction of the number of pentagon arms by increasing the incident angle in fact the size of the particles interacting with the $s$-polarized light is decreased, hence causing blue shift. In addition, the incident light may not be able to interact with the bending joint (hot spot) of the top and the underlying arms.

Considering the results of $p$-polarization for $10^{\circ}$ incident angle (Figure 4(a)) the order of the intensity of the peak in relation to the $\varphi$ angle is $45^{\circ}, 0^{\circ}$ and $180^{\circ}, 90^{\circ}$, and $135^{\circ}$. This order is similar to the $s$-polarized spectra discussed above. 


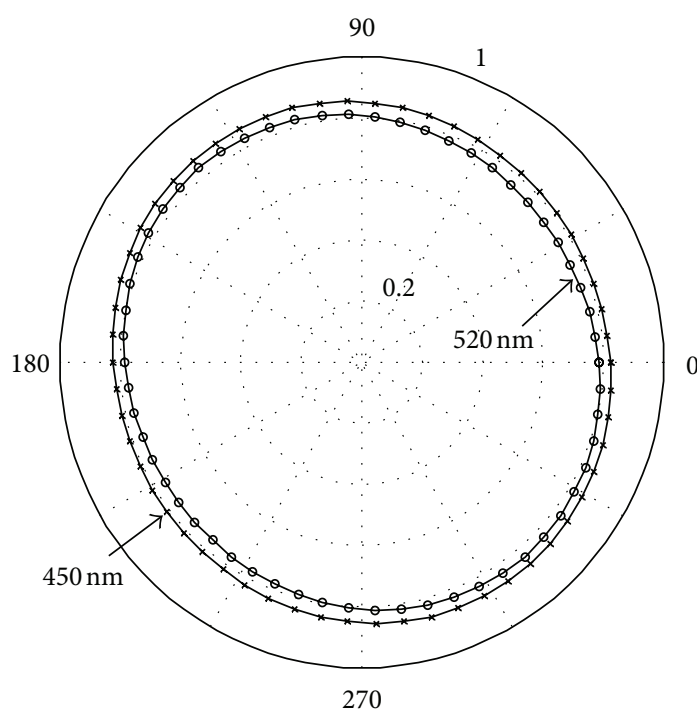

(a)

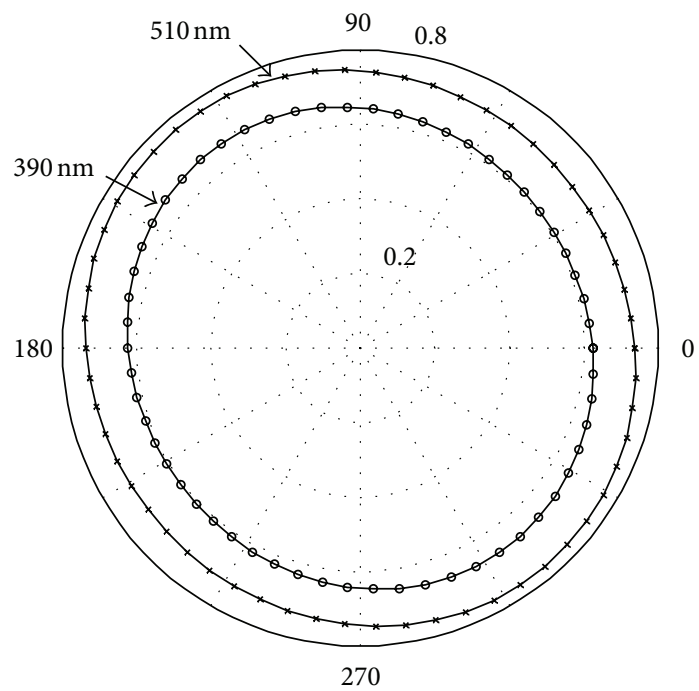

(c)

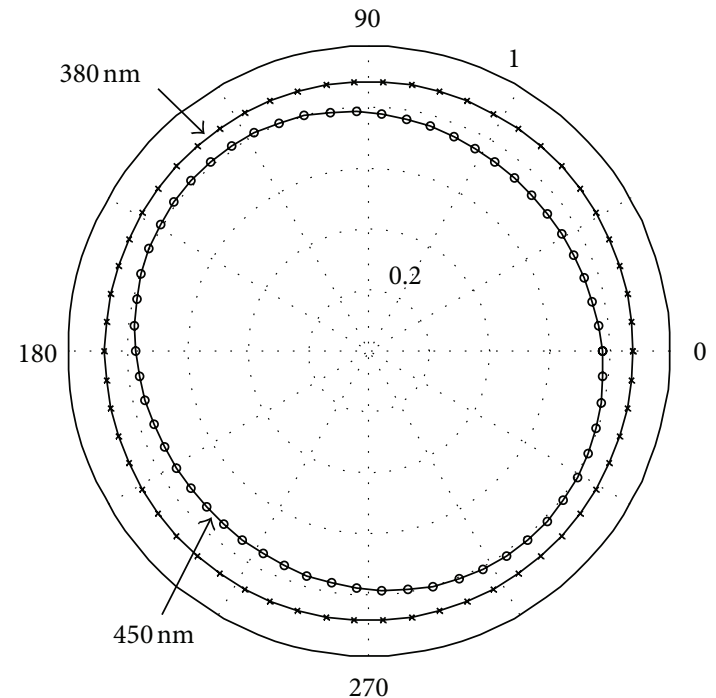

(b)

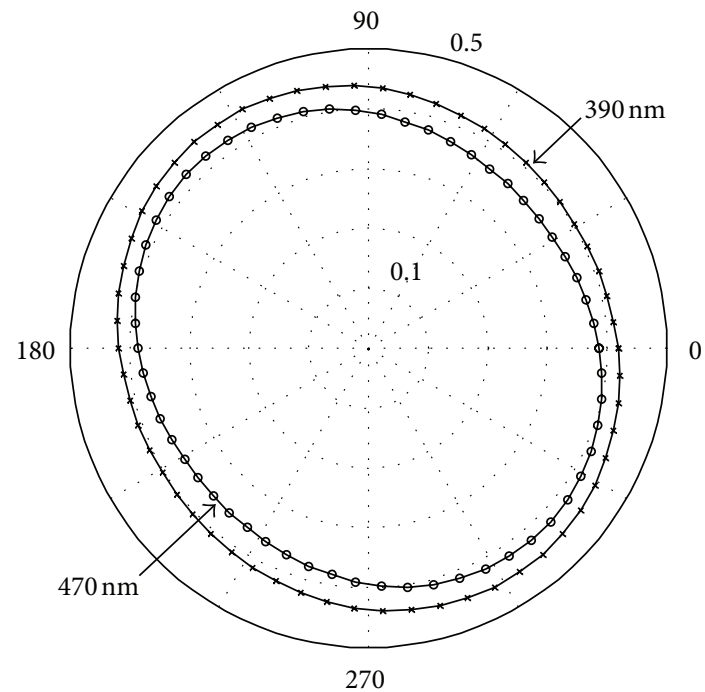

(d)

Figure 5: Polar plots of the $(1-R)$ spectra of Ag chiral pentagon thin film for TM and LM wavelengths obtained at two different incident angles $(\theta)$. ((a) and (b)) $\theta=10^{\circ}, p$ - and $s$-polarizations, respectively; ((c) and (d)) $\theta=70^{\circ}, p$ - and $s$-polarizations, respectively.

However, for $70^{\circ}$ incident angle (Figure 4(c)) the order is as follows: $135^{\circ}, 90^{\circ}, 0^{\circ}, 180^{\circ}$, and $45^{\circ}$, which is opposite to that of $10^{\circ}$ incident angle. Therefore, it can be concluded that at the lowest incident angle the $p$-polarized light interacts with the pentagon structure almost similar to that of $s$-polarized light. This is because at $10^{\circ}$ incident angle the $E_{p \|}$ component of $E_{p}$ field is small and has no considerable effect on the absorption spectra, while by increasing the incident angle to $70^{\circ}$ this component increases in size and influences the spectra. The intriguing feature in the $70^{\circ}$ incident angle of p-polarized light (Figure 4(c)) is the considerable increase of absorption at wavelengths greater than $550 \mathrm{~nm}$ which is due to the increased $E_{p \|}$ component of $E_{p}$ field. This property of pentagon sculptured thin film at high incident angle for p-polarized light, particularly at infrared (IR) wavelengths $(>800 \mathrm{~nm})$ may be used as a useful probe in killing of cancer cells with photothermal therapy in near infrared region [51].

\subsubsection{Polarization Dependent Optical Spectra of Silver Helical} Pentagon Thin Film. As mentioned above, the polarization dependent spectra should confirm the extent of existence of anisotropy in the structure, if such anisotropy exists at all. Therefore, using the data presented in Section 3.2.1, we plotted their polar diagrams in order to achieve more insight into this issue, though our azimuthal angle measurements were carried out at $45^{\circ}$ intervals/steps. More quantitative data could be achieved if smaller azimuthal angle intervals had been measured. 


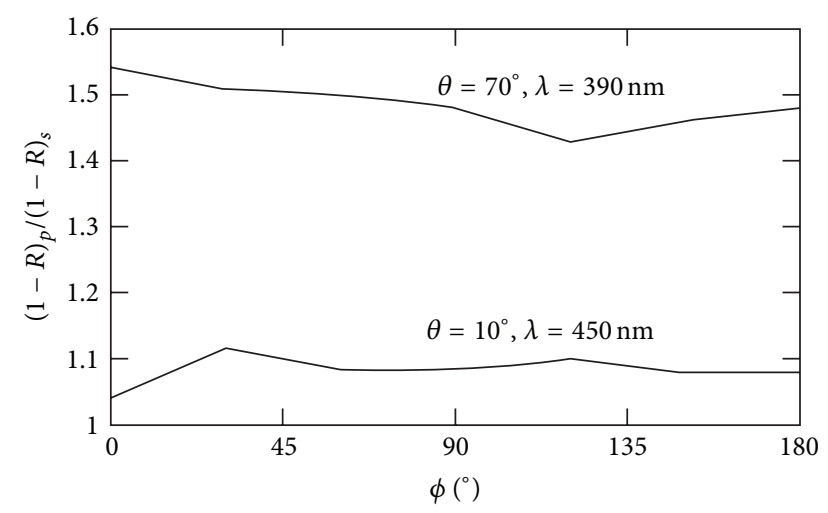

FIgURE 6: The polarization $(1-R)$ ratio $(1-R)_{p} /(1-R)_{s}$ versus azimuthal angle for two incident light angles of $10^{\circ}$ and $70^{\circ}$ on helical pentagon shaped Ag sculptured thin film.

Figures 5(a) and 5(b) show the angular dependent spectra of the silver chiral pentagon for both $p$ - and $s$-polarized incident lights at low incidence angle of $10^{\circ}$ and for different wavelengths.

From these polar figures it can be deduced that at these low incidence angles a high degree of anisotropy in the extinction spectra of the silver helical pentagon sculptured thin film is not observed. The slight anisotropy may confirm the above discussion and the results given in Table 2 .

However, results for $70^{\circ}$ incidence angle (Figures 5(c) and $5(\mathrm{~d})$ ) show some degree of anisotropy. This is again in agreement with the discussion given in Section 3.2.

Since the resemblance of a structure with 5-fold symmetry (e.g., pentagon helical structure) to a circular chiral structure is high one should not expect to observe a high degree of anisotropy. However, our results in Figure 5 indicate that even this slight anisotropy can be distinguished, using the rough procedure carried out in this work which could be due to the top layer of the film.

Zhao et al. [52] have discussed the anisotropy of Ag nanorod arrays prepared by oblique angle deposition on the basis of Gans theory for spheroid particles [53] and concluded that the absorbance ratio $\left(\alpha_{p} / \alpha_{s}\right)$ at $p$ - and $s$-polarizations reflects the anisotropic nature of the nanorods. If $\alpha_{p} / \alpha_{s}>1$, the nanorods have a long axis approximately along the $p$ polarization direction; if $\alpha_{p} / \alpha_{s}<1$, the nanorods have a long axis approximately along the $s$-polarization direction. Considering that each of our helical pentagons is an assembly of nanorods we may apply this procedure to our results given in Figures 4 and 5 and may achieve a conclusion about the anisotropy of our $(1-R)$ spectra detected at different incident light angles. Figure 6 shows this ratio (i.e., $(1-R)_{p} /(1-R)_{s}$ ) for incident angle of $10^{\circ}$ at the wavelength of $450 \mathrm{~nm}$ and incident angle of $70^{\circ}$ at the wavelength of $390 \mathrm{~nm}$ as a function of azimuthal angle, as typical examples. It can be seen that in both cases the pentagons arranged of nanorods have their long axis along the $p$-polarization direction while this is more pronounced at larger incidence angles. These results are consistent with our earlier discussion in Section 3.2.1.

\section{Conclusions}

Optical spectra of silver helical pentagon shaped nanosculptured thin films, produced using $\mathrm{OAD}$, together with rotation of substrate holder were investigated using a single beam spectrophotometer with both $s$ - and $p$-polarization incident light at $10^{\circ}$ and $70^{\circ}$ incidence angles and at different azimuthal angles. No obvious change in the position of peak in the extinction spectra obtained at low incidence angles with $s$-polarization light was observed, while at $70^{\circ}$ incidence angle a blue shift was distinguished. $p$-polarized light at $70^{\circ}$ incidence angle caused a red shift in the extinction spectrum. Morphology and nanostructure of the films were obtained by means of AFM and FESEM.

\section{Conflict of Interests}

The authors declare that there is no conflict of interests regarding the publication of this paper.

\section{Acknowledgments}

This work was carried out with the support of the University of Tehran. Hadi Savaloni is grateful to the Iran National Science Foundation (INSF) and the Centre of Excellence for Physics of Structure and Microscopic Properties of Matter, Department of Physics, University of Tehran, for partial support of this work.

\section{References}

[1] K. Robbie, G. Beydaghyan, T. Brown, C. Dean, J. Adams, and C. Buzea, "Ultrahigh vacuum glancing angle deposition system for thin films with controlled three-dimensional nanoscale structure," Review of Scientific Instruments, vol. 75, no. 4, pp. 1089-1097, 2004.

[2] B. N. Khlebtsov, V. A. Khanadeyev, J. Ye, D. W. Mackowski, G. Borghs, and N. G. Khlebtsov, "Coupled plasmon resonances in monolayers of metal nanoparticles and nanoshells," Physical Review B, vol. 77, no. 3, Article ID 035440, 2008.

[3] P. K. Jain, K. S. Lee, I. H. El-Sayed, and M. A. El-Sayed, “Calculated absorption and scattering properties of gold nanoparticles of different size, shape, and composition: applications in biological imaging and biomedicine," Journal of Physical Chemistry B, vol. 110, no. 14, pp. 7238-7248, 2006.

[4] J. C. Riboh, A. J. Haes, A. D. McFarland, C. R. Yonzon, and R. P. Van Duyne, "A nanoscale optical biosensor: real-time immunoassay in physiological buffer enabled by improved nanoparticle adhesion," Journal of Physical Chemistry B, vol. 107, no. 8, pp. 1772-1780, 2003.

[5] K. E. Shafer-Peltier, C. L. Haynes, M. R. Glucksberg, and R. P. Van Duyne, "Toward a glucose biosensor based on surfaceenhanced Raman scattering," Journal of the American Chemical Society, vol. 125, no. 2, pp. 588-593, 2003.

[6] N. L. Rosi and C. A. Mirkin, "Nanostructures in biodiagnostics," Chemical Reviews, vol. 105, no. 4, pp. 1547-1562, 2005.

[7] S. Pal, Y. K. Tak, and J. M. Song, "Does the antibacterial activity of silver nanoparticles depend on the shape of the nanoparticle? A study of the gram-negative bacterium Escherichia coli," 
Applied and Environmental Microbiology, vol. 73, no. 6, pp. 17121720, 2007.

[8] M. Suzuki and Y. Taga, "Integrated sculptured thin films," Japanese Journal of Applied Physics, Part 2, vol. 40, no. 4 A, pp. L358-L359, 2001.

[9] Q. Wu, I. J. Hodgkinson, and A. Lakhtakia, "Circular polarization filters made of chiral sculptured thin films: experimental and simulation results," Optical Engineering, vol. 39, no. 7, pp. 1863-1868, 2000.

[10] S. A. Maier, P. G. Kik, H. A. Atwater et al., "Local detection of electromagnetic energy transport below the diffraction limit in metal nanoparticle plasmon waveguides," Nature Materials, vol. 2, no. 4, pp. 229-232, 2003.

[11] J. Z. Zhang and C. Noguez, "Plasmonic optical properties and applications of metal nanostructures," Plasmonics, vol. 3, no. 4, pp. 127-150, 2008.

[12] I. J. Hodgkinson, A. Lakhtakia, Q. H. Wu, L. De Silva, and M. W. McCall, "Ambichiral, equichiral and finely chiral layered structures," Optics Communications, vol. 239, no. 4-6, pp. 353358, 2004.

[13] A. C. Van Popta, M. J. Brett, and J. C. Sit, "Double-handed circular Bragg phenomena in polygonal helix thin films," Journal of Applied Physics, vol. 98, no. 8, Article ID 083517, 2005.

[14] M. Dixit and A. Lakhtakia, "Selection strategy for circularpolarization-sensitive rejection characteristics of electro-optic ambichiral Reusch piles," Optics Communications, vol. 281, no. 19, pp. 4812-4823, 2008.

[15] S. R. Kennedy, M. J. Brett, O. Toader, and S. John, "Fabrication of tetragonal square spiral photonic crystals," Nano Letters, vol. 2, no. 1, pp. 59-62, 2002.

[16] R. Abdeddaim, G. Guida, A. Priou, B. Gallas, and J. Rivory, "Negative permittivity and permeability of gold square nanospirals," Applied Physics Letters, vol. 94, no. 8, Article ID 081907, 2009.

[17] K. Robbie and M. J. Brett, "Sculptured thin films and glancing angle deposition: growth mechanics and applications," Journal of Vacuum Science and Technology A, vol. 15, no. 3, pp. 1460$1465,1997$.

[18] A. P. Alivisatos, "Semiconductor clusters, nanocrystals, and quantum dots," Science, vol. 271, no. 5251, pp. 933-937, 1996.

[19] S. Link and M. A. El-Sayed, "Spectral properties and relaxation dynamics of surface plasmon electronic Oscillations in gold and silver nanodots and nanorods," Journal of Physical Chemistry B, vol. 103, no. 40, pp. 8410-8426, 1999.

[20] S. Chen, S. Webster, R. Czerw, J. Xu, and D. L. Carroll, "Morphology effects on the optical properties of silver nanoparticles," Journal of Nanoscience and Nanotechnology, vol. 4, no. 3, pp. 254-259, 2004.

[21] S. A. Harfenist, Z. L. Wang, R. L. Whetten, I. Vezmar, and M. M. Alvarez, "Three-dimensional hexagonal close-packed superlattice of passivated Ag nanocrystals," Advanced Materials, vol. 9, no. 10, pp. 817-822, 1997.

[22] P. Orfanides, T. F. Buckner, M. C. Buncick, F. Meriaudeau, and T. L. Ferrell, "Demonstration of surface plasmons in metal island films and the effect of the surrounding medium-an undergraduate experiment," American Journal of Physics, vol. 68, no. 10, pp. 936-942, 2000.

[23] G. Xu, M. Tazawa, P. Jin, S. Nakao, and K. Yoshimura, "Wavelength tuning of surface plasmon resonance using dielectric layers on silver island films," Applied Physics Letters, vol. 82, no. 22, pp. 3811-3813, 2003.
[24] J. J. Mock, D. R. Smith, and S. Schultz, "Local refractive index dependence of plasmon resonance spectra from individual nanoparticles," Nano Letters, vol. 3, no. 4, pp. 485-491, 2003.

[25] C. L. Nehl, H. Liao, and J. H. Hafner, "Optical properties of starshaped gold nanoparticles," Nano Letters, vol. 6, no. 4, pp. 683$688,2006$.

[26] S. B. Chaney, Z.-Y. Zhang, and Y.-P. Zhao, "Anomalous polarized absorbance spectra of aligned Ag nanorod arrays," Applied Physics Letters, vol. 89, no. 5, Article ID 053117, 2006.

[27] Z. Zhang and Y. Zhao, "Optical properties of U-shaped Ag nanostructures," Journal of Physics Condensed Matter, vol. 20, no. 34, Article ID 345223, 2008.

[28] Z.-Y. Zhang and Y.-P. Zhao, "Tuning the optical absorption properties of Ag nanorods by their topologic shapes: a discrete dipole approximation calculation," Applied Physics Letters, vol. 89, no. 2, Article ID 023110, 2006.

[29] L. Eckertova, Physics of Thin Films, chapter 1, Plenum Press, 2nd edition, 1990.

[30] H. Savaloni, F. Babaei, S. Song, and F. Placido, "Characteristics of sculptured $\mathrm{Cu}$ thin films and their optical properties as a function of deposition rate," Applied Surface Science, vol. 255, no. 18, pp. 8041-8047, 2009.

[31] L. Abelmann and C. Lodder, "Oblique evaporation and surface diffusion," Thin Solid Films, vol. 305, no. 1-2, pp. 1-21, 1997.

[32] M. G. Shahraki and H. Savaloni, "Thin-film-growth characteristics by computer simulation: nanostructural changes as a function of deposition conditions," Physica Status Solidi (B), vol. 244, no. 9, pp. 3115-3136, 2007.

[33] H. Savaloni and M. G. Shahraki, "A computer model for the growth of thin films in a structure zone model," Nanotechnology, vol. 15, no. 3, pp. 311-319, 2004.

[34] Q. Zhou, Y. He, J. Abell, Z. Zhang, and Y. Zhao, "Surfaceenhanced Raman scattering from helical silver nanorod arrays," Chemical Communications, vol. 47, no. 15, pp. 4466-4468, 2011.

[35] H. Savaloni and R. Babaei, "Surface enhanced Raman spectroscopy and structural characterization of $\mathrm{Ag} / \mathrm{Cu}$ chiral nanoflower sculptured thin films," Applied Surface Science, vol. 280, no. 1, pp. 439-445, 2013.

[36] U. Keibig and M. Vollmer, Optical Properties of Metal Clusters, Springer, Berlin, Germany, 1995.

[37] O. S. Heavens, Optical Properties of Thin Solid Films, Butterworths, London, UK, 1955.

[38] A. Lakhtakia, "On percolation and circular Bragg phenomenon in metallic, helicoidally periodic, sculptured thin films," Microwave Optical Technology Letters, vol. 24, no. 4, pp. 239242, 2000.

[39] A. Siabi-Garjan and H. Savaloni, "Extinction spectra and electric field enhancement of silver chiral nano-flower shaped nanoparticle, Comparison of discrete dipole approximation results with experimental results," European Physical Journal B, vol. 86, no. 257, p. 30943, 2013.

[40] B. Dick, M. J. Brett, and T. Smy, "Controlled growth of periodic pillars by glancing angle deposition," Journal of Vacuum Science and Technology B, vol. 21, no. 1, pp. 23-28, 2003.

[41] B. Dick, M. J. Brett, and T. Smy, "Investigation of substrate rotation at glancing incidence on thin-film morphology," Journal of Vacuum Science and Technology B, vol. 21, no. 6, pp. 2569-2575, 2003.

[42] H. Savaloni, F. Babaei, S. Song, and F. Placido, "Influence of substrate rotation speed on the nanostructure of sculptured $\mathrm{Cu}$ thin films," Vacuum, vol. 85, no. 7, pp. 776-781, 2011. 
[43] C. F. Bohren and D. R. Huffman, Absorption and Scattering of Light by Small Particles, John Wiley \& Sons, New York, NY, USA, 1983.

[44] H. Savaloni, F. Haydari-Nasab, and M. Malmir, "Nano-structural characteristics and optical properties of silver chiral nanoflower sculptured thin films," Applied Surface Science, vol. 257, no. 21, pp. 9044-9055, 2011.

[45] F. Abdi, A. Siabi-Gerjan, and H. Savaloni, "On the discrete dipole approximation investigation of extinction spectra of $\mathrm{Ag}$ /glass nano-flower thin film with threefold symmetry," Journal of Theoretical and Applied Physics, vol. 6, no. 4, pp. 1-10, 2012.

[46] F. Abdi, A. Siabi-Gerjan, and H. Savaloni, "Investigation on the dependence of optical spectra of silver chiral nanostructures on shape, dimensions and incident light by discrete dipole approximation," Journal of Theoretical and Applied Physics, vol. 6, no. 1, pp. 1-7, 2012.

[47] A. Siabi-Garjan, H. Savaloni, F. Abdi, A. Ghaffal, and F. Placido, "Investigation on the optical properties of $\mathrm{ZnS}$ nano-rod and chiral sculptured thin films using experimental and theoretical approaches," Physica Scripta, vol. 87, no. 5, Article ID 055705, 2013.

[48] J. D. Jackson, Classical Electrodynamics, John Wiley \& Sons, New York, NY, USA, 2nd edition, 1975.

[49] J. R. Reitz and F. J. Milford, Foundations of Electromagnetic Theory, Addison-Wesley, 1960.

[50] E. D. Palik, Handbook of Optical Constants of Solids, Academic Press, New York, NY, USA, 1985.

[51] X. Huang, I. H. El-Sayed, W. Qian, and M. A. El-Sayed, "Cancer cell imaging and photothermal therapy in the nearinfrared region by using gold nanorods," Journal of the American Chemical Society, vol. 128, no. 6, pp. 2115-2120, 2006.

[52] Y.-P. Zhao, S. B. Chaney, and Z.-Y. Zhang, "Absorbance spectra of aligned Ag nanorod arrays prepared by oblique angle deposition," Journal of Applied Physics, vol. 100, no. 6, Article ID 063527, 2006.

[53] S. Link, M. B. Mohamed, and M. A. El-Sayed, "Simulation of the optical absorption spectra of gold nanorods as a function of their aspect ratio and the effect of the medium dielectric constant," Journal of Physical Chemistry B, vol. 103, no. 16, pp. 3073-3077, 1999. 

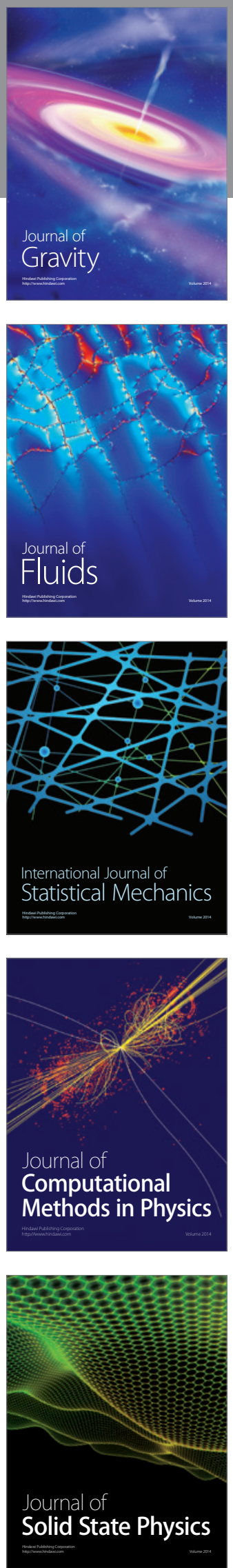

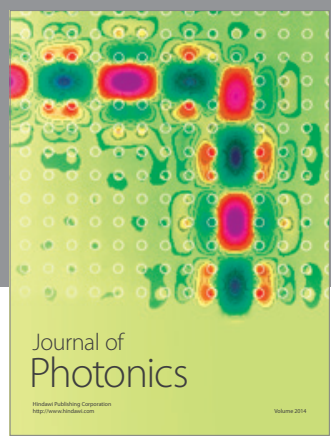

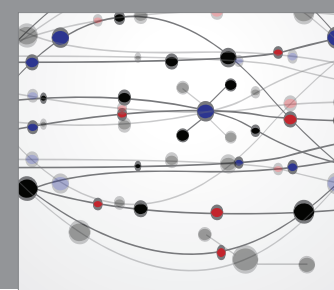

The Scientific World Journal

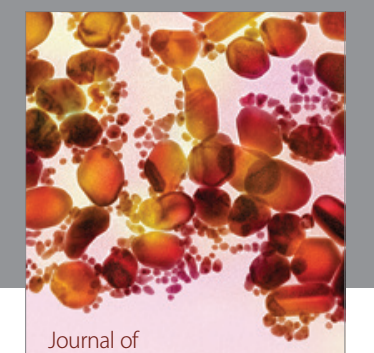

Soft Matter
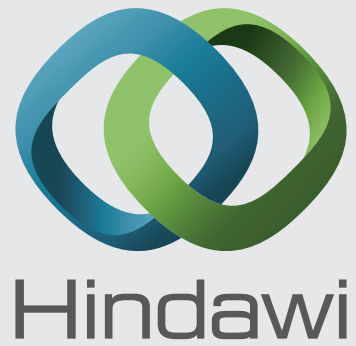

Submit your manuscripts at

http://www.hindawi.com
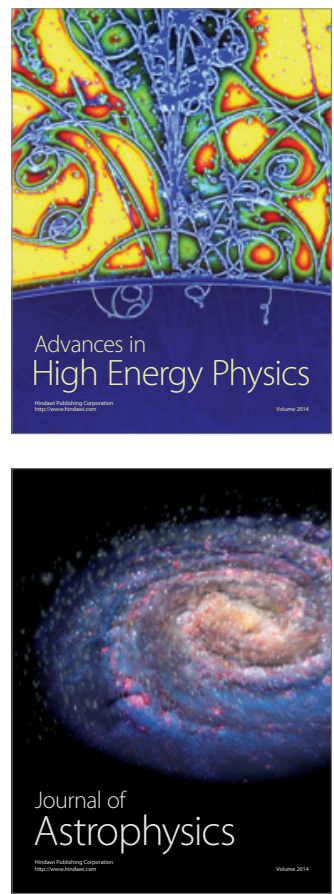
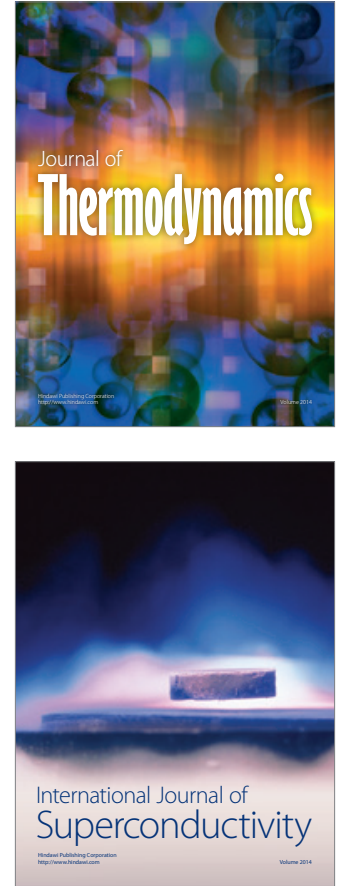
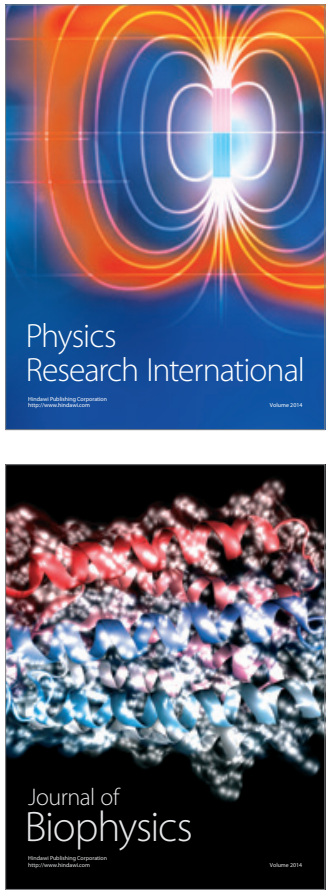
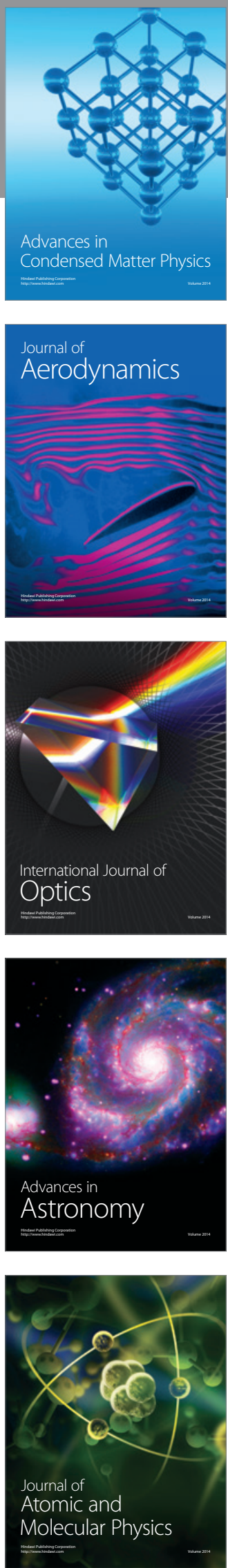